\title{
CASE REPORT: METASTASIS IN THE RADIUS 6 YEARS AFTER THE TREATMENT OF INVASIVE BREAST CARCINOMA
}

Ridania de Oliveira Frederice ${ }^{1}$

${ }^{1}$ Hospital Sírio-Libanês - São Paulo (SP), Brazil.

Introduction: It is estimated that $6 \%$ of the women with breast cancer $(\mathrm{BC})$ present metastasis at diagnosis. In this context, the bone is the main site, followed by lung, liver and central nervous system. IN 15\% of them, the bone presents as a single metastasis site, especially in the axial skeleton; unfrequently in the pelvis, and rarely in the appendicular skeleton. Case Report: In 2009, a female, 53-year old patient, in the menopause, hypertensive and former smoker, without family history of cancer, presented a palpable nodule in the right breast. At a consultation in the social responsibility service of Hospital Sírio Libanês, she presented a fragment biopsy compatible with invasive breast SOE, GH2, GN3, RE 80\%, RP 50\% and Ki67 20\%. Staging was cT2 cN3C cM0. She underwent neoadjuvant chemotherapy with 4 cycles of doxorubicin with cyclophosphamide, and 12 of paclitaxel. She underwent quadrantectomy in the right breast, ipsilateral axillary lymphadenectomy, and reconstruction with thoracodorsal flap in August, 2010. Anatomopathological pT2pN3. She underwent radiotherapy of the breast and drainage, including supraclavicular fossa (50Gy) and boos (10Gy) until October, 2010. She used adjuvant anastrozole. In March, 2016, she reports pain and sudden loss of strength in the right forearm. X-ray and tomography showed extensive lesion in the radius, associated with pathological fracture without joint impairment. Systemic staging without other disease sites. Even though it was not possible to perform the biopsy, after a solid radiological evaluation, a secondary lesion was considered. She was started on exemestane and zoledronic acid, associated with immobilization and palliative radiotherapy (30Gy). The patient was asymptomatic, presented with pulmonary and lymph node progression in January, 201, changing from endocrine therapy to tamoxifen. In December, 2019, she started with fulvestrant de to discreet lymph node progression. She has been followed-up by an oncologist. Discussion: Lately, with advances in the multimodal treatment of BC, the global survival (GS) of the disease in stage IV has been increasing. Patients with single bone metastasis (SBM) have better prognosis, being mostly luminal tumors treated with the change of endocrine therapy. The main site of implantation is the lumbar and thoracic spine, and very rarely, the radius. Lee et al. reported, among 146 patients with bone metastasis, that 26 were in the femur; 5 , in the humerus, and none in the radius, with progression-free survival of 24 months, and GS of 79 months. BC metastasis with appendicular skeleton, especially in the radius, are very rare; however, when SBM, present with better prognosis. 